\title{
Ökotoxikologische Prüfungen nach dem Chemikaliengesetz (ChemG)
}

\section{- Durchführung und Bewertung}

\author{
H. Peter, C. Franke \\ Dr. H. Peter, Dr. C. Franke, Umweltbundesamt, Mauerstraße 52, D-O-1080 Berlin
}

Zusammenfassung. Das Chemikaliengesetz schreibt für neu anzumeldende Stoffe, nach Vermarktungsmengen gestaffelt, ökotoxikologische Prüfungen vor, deren Ergebnisse eine Abschätzung des Gefährdungspotentials für die Umwelt ermöglichen. Die ökotoxikologischen Tests - deren Prüfung auf Validität und deren Bewertung dem Umweltbundesamt obliegen - werden beschrieben. Darüber hinaus werden Hinweise für die Testauswahl, für Problemlösungen bei der Versuchsdurchführung und für die Auswertung der Ergebnisse gegeben.

\section{Einleitung}

Die ökotoxikologische Bewertung chemischer Stoffe basiert auf Erkenntnissen hinsichtlich ihrer Toxizität, biologischen Abbaubarkeit und Bioakkumulation. Zu diesen Bereichen sind nach dem Chemikaliengesetz gemäß Anhang V - in Verbindung mit den Anhängen VII und VIII - der Richtlinie 67/548/EWG des Rates vom 27. Juni 1967 (6. Änderungs-Richtlinie) bestimmte Tests durchzuführen. Die Tests sind für

- die Grundstufe im Amtsblatt der EWG L 251 vom 19. September 1984 (Richtlinie 84/449/EWG),

- die Stufe 1 im Amtsblatt der EWG L 133/1 vom 30. Mai 1988 (Richtlinie 87/302/EWG)

veröffentlicht. Tests nach anderen Prüfrichtlinien - z.B. die der OECD - werden akzeptiert, wenn sie im wesentlichen den EG-Prüfrichtlinien entsprechen oder wenn die EG-Prüfrichtlinien nicht anwendbar sind.

\section{Toxizität}

\subsection{Toxizitätstests der Grundstufe}

\subsubsection{Aquatische Toxizität}

\section{Akute Toxizität für Fische}

(Anhang V. C.1, überarbeitete Fassung)

Ziel des Tests ist die Ermittlung der akuten letalen Toxizität des zu prüfenden Stoffes für Fische.
Diese Toxizität wird in Form des LC50-Wertes angegeben, das ist die Konzentration im Wasser, die $50 \%$ der Testfische innerhalb einer definierten Zeit tötet (letale Konzentration). Ein Testfisch wird als tot registriert, wenn er bei Berührung des Schwanzansatzes keine Reaktion zeigt und keine Atembewegungen erkennbar sind.

Empfehlungen für die Auswahl der Testspezies finden sich in der Prüfvorschrift; sehr häufig wird wegen seiner problemlosen Hälterung in Deutschland der Zebrabärbling verwendet. Es werden mindestens 5 Prüfkonzentrationen angesetzt, die sich durch einen konstanten Faktor von maximal 2,2 unterscheiden, für jede Konzentration sind mindestens 7 Fische einzusetzen. Ein Beispiel für eine Konzentrations-Wirkungs-Kurve zeigt Abb. $1(\rightarrow A b b .1)$.

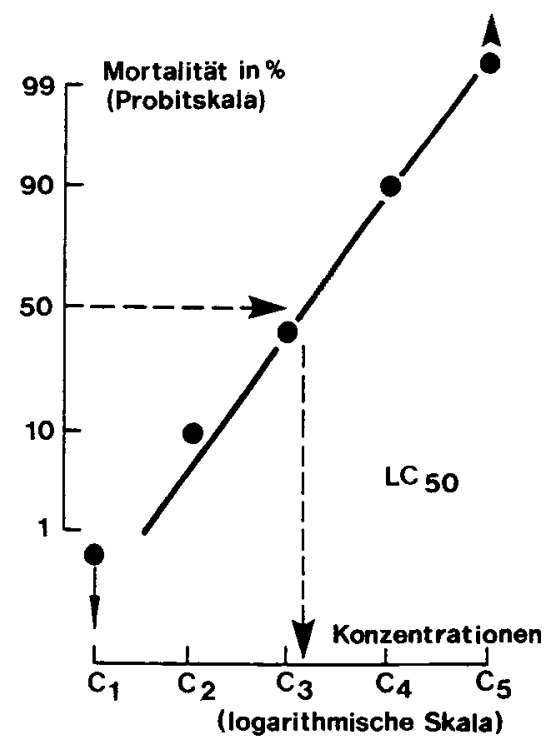

Abb. 1: Konzentrations-Wirkungs-Kurve

Der aus dieser Kurve abzulesende LC50-Wert für 96 Stunden dient als Grundlage der Bewertung - auch zur Festlegung des Gefährlichkeitsmerkmals "umweltgefährlich * (R-Satz) - und wird allen EG-Staaten übermittelt, ebenso wie die Grunddaten der übrigen Tests. Der LC50-Wert kann jedoch lediglich einen Anhaltspunkt für die akute Fischgiftigkeit des zu testenden Stoffes geben; zur genaue- 
ren Bewertung, muß der Verlauf der KonzentrationsMortalitäts-Kurve interpretiert werden. Ein sehr flacher Kurvenverlauf indiziert beginnende letale Effekte (LC16-Werte) bereits bei sehr geringen Stoffkonzentrationen, so daß eine Gefährdung schon bei Konzentrationen weit unterhalb des LC50-Wertes möglich ist. Bei einem sehr steilen Kurvenverlauf sind geringe Stoffkonzentrationen nicht effektiv, jedoch führen bereits Konzentrationen knapp über dem LC50-Wert zur Letalität der gesamten Fischpopulation. Zur Charakterisierung des gesamten Kurvenverlaufs ist es daher sinnvoll, neben den LC50-Werten den LC0- und den LC100 (96 Std.)-Wert anzugeben.

Der zeitliche Verlauf der toxischen Wirkung ist anhand der LC50-Werte für 24, 48, 72 und 96 Stunden abzulesen. Bei einer starken Zeitabhängigkeit der Intoxikation innerhalb des Meßzeitraums von 96 Stunden lassen sich erhöhte Toxizitäten bei längerfristiger und chronischer Exposition vermuten, im Gegensatz zu einer geringen oder nicht vorhandenen Zeitabhängigkeit der Vergiftungen.

Ein wichtiges Qualitätskriterium für die Validität des Fischtests und der übrigen Tests zur aquatischen Toxizität ist die Aufrechterhaltung einer ausreichenden Konzentration der Prüfsubstanz, die während des gesamten Prüfzeitraums um nicht mehr als $20 \%$ abnehmen darf.

Die Einhaltung dieser Bedingung bereitet keine Schwierigkeiten bei Substanzen, die leicht wasserlöslich und stabil sind und die sich weder verflüchtigen, noch an den Wandungen der Versuchsgefäße adsorbiert werden.

Im Falle schwer löslicher, leicht flüchtiger und/oder instabiler Substanzen oder Substanzen, die stabile Suspensionen oder Dispersionen bilden, soll die Anfangskonzentration nach einer Zeit der Äquilibrierung analytisch bestimmt werden und zumindest am Ende des Tests eine weitere Analyse erfolgen.

Wird im statischen Test eine Konzentrationsabnahme um mehr als $20 \%$ erwartet oder gemessen, so sollte ein semistatischer oder ein Durchfluß-Test erwogen werden. In Einzelfällen kann es geschehen, daß selbst bei Wahl der semistatischen oder Durchfluß-Methode die Qualitätskriterien bezüglich der Konzentrations-Konstanz nicht erfüllt werden können. In diesen Fällen ist es möglich, die LCWerte auf die gemittelten analytisch bestimmten Realkonzentrationen zu beziehen, wie es in der OECD-Testguideline 203 beschrieben wird (1981, revidiert 1983; OECD Council Adoption of Draft Guidelines for Testing of Chemicals C(84)32). Bei sehr schnell zerfallenden Stoffen kann auch ein Test mit dem Zerfallsprodukt oder den Zerfallsprodukten sinnvoll sein.

Schwer lösliche Substanzen können mit Hilfe von Lösungsvermittlern wie Aceton oder Tween in Lösung gebracht werden, doch darf die Konzentration der Hilfsstoffe nicht mehr als $100 \mathrm{mg} / \mathrm{l}$ betragen. Schwierigkeiten können sich bei der Interpretation der LC-Daten ergeben, wenn die mit Lösungsvermittlern erzielten Werte um Größenordnungen höher liegen als es der ,,normalen“'Wasserlöslichkeit des Stoffes entspricht. Daher wird empfohlen, in einem LimitTest die Substanzen nur bis zur Löslichkeitsgrenze ohne Hilfsstoffe zu testen oder bis zu der höchsten Konzentration, die eine stabile Dispersion bildet. Werden im Bereich der Löslichkeitsgrenze keine letalen Effekte beobachtet, so ist eine LC50 > Löslichkeitsgrenze anzugeben.

Für lösliche Substanzen kann ein Limit-Test mit einer Konzentration von $100 \mathrm{mg} / 1$ durchgeführt werden.

\section{Akute Toxizität für Daphnien}

(Anhang V, C.2, überarbeitete Fassung)

Die akute Daphniengiftigkeit der zu testenden Substanz wird durch den EC50-Wert angegeben; das ist diejenige mittlere effektive (wirksame) Konzentration, die innerhalb einer definierten Zeit bei $50 \%$ der Versuchstiere eine Schwimmunfähigkeit bewirkt. Als schwimmunfähig gelten Daphnien, die nach leichter Bewegung des Prüfbehälters innerhalb von 15 Sekunden keine Schwimmbewegungen zeigen. Der Versuchszeitraum erstreckt sich über 48 Stunden. Bestimmt werden die EC50-Werte nach 24 und 48 Stunden; die ECO- und EC100-Werte nach 48 Stunden sollten ebenfalls angegeben werden. Die Qualitätskriterien bezüglich der Aufrechterhaltung der Prüfkonzentration während des Meßzeitraums sind im Prinzip dieselben wie beim akuten Fischtest; dasselbe gilt für die Kriterien des Limit-Tests.

\subsubsection{Terrestrische Toxizität}

In der Grundstufe werden keine spezifischen Tests zur terrestrischen Ölkotoxikologie verlangt, jedoch können die an Nagern durchgeführten Tests Hinweise auf mögliche Gefährdungen terrestrischer Lebewesen geben. Besonders die Resultate aus dem Test zur subakuten Toxizität an Ratten bei oraler Aufnahme werden auf eine Verwertbarkeit zum Abschätzen eines Umweltgefährdungspotentials überprüft. Darüber hinaus geben die Tests zur Mutagenität Anhaltspunkte für ein mögliches kanzerogenes Potential bei terrestrischen, aber auch bei aquatischen Lebewesen.

\subsection{Toxizitätstests der Stufe I}

\subsubsection{Aquatische Toxizität}

\section{Der längerfristige Fischtest}

\section{(1) Nach OECD 204}

Dieser Test ist in erster Linie als Letalitätstest konzipiert; die Ergebnisse werden in Form des LC50-Wertes angegeben. Die anzugebende NOEC (No Effect Concentration) bezieht sich auf Symptome im subletalen Bereich. Diese Symptome, z.B. Veränderungen des Schwimmverhaltens oder Pigmentierungen, sind zwar für die Bewertung der toxischen Wirkung der Testsubstanz sehr ernst zu nehmen, da sie in der freien Natur sehr häufig den Tod des Fisches zur Folge haben, doch lassen sie sich im Test schwer quantifizieren. Wenig sinnvoll ist die Durchführung des verlängerten Fischtests, wenn sich im akuten Fischtest während eines Zeitraums von 96 Stunden keine Letalitätssteigerung ergibt, so daß auch weitere letale Effekte in einem Zeitraum von 14-28 Tagen nicht wahrscheinlich sind. In diesem Fall wird die Durchführung eines Early-Life-Stage-Tests am Fisch empfohlen. 


\section{(2) Early-Life-Stage-Test (Fisch) (OECD, Entwurf)}

Der Early-Life-Stage-Test verläuft über ca. 4 Wochen und erfüllt somit die Kriterien der Richtlinie 67/548/EWG des Rates vom 27. Juni 1967 über einen längerfristigen Fischtest. Er kann als Teil eines Full-Life-Cycle-Tests angesehen werden und liefert vielfältige Informationen, da er die Embryo- und Larvalentwicklung sowie die frühe Wachstumsphase des Fisches einbezieht. Aus diesem Grunde ist der Test dem längerfristigen Test mit alleiniger Letalität als Endpunkt vorzuziehen. Zu bestimmen sind die NOEC (No Effect Concentration) sowie die LOEC (Lowest Effect Concentration), bezogen auf Parameter wie

- Letalität (Überlebensrate von Eiern, Larven und Jung. fischen),

- Zeitpunkt des Schlüpfens,

- Anzahl der geschlüpften Tiere,

- morphologische Abnormitäten.

\section{Verlängerte Daphnien-Toxizität (OECD 202, Part II)}

Grundlage für die toxikologische Bewertung der Testsubstanz ist in der Regel der auf die Reproduktion der Daphnien bezogene NOEC-Wert. Diese NOEC berücksichtigt in erster Linie eine Verringerung der Nachkommenzahl pro Elterntier nach 21 Tagen, aber auch zeitliche Verschiebungen der Geburt sowie Wachstumsverzögerungen der Nachkommen. In manchen Fällen sind jedoch andere Parameter empfindlicher, wie z.B. die Mortalität der Elterntiere. Aus diesem Grund ist es unbedingt notwendig, alle biologischen Effekte zu registrieren und einen auf den empfindlichsten Parameter bezogenen NOEC-Wert zu bestimmen.

\section{Wachstumshemmung an der Alge}

(Anhang V, C. 3, überarbeitete Fassung)

Mit diesem Test wird die Wirkung einer Substanz auf das Wachstum einer einzelligen Grünalgenart bestimmt. Der Test erstreckt sich über 72 Stunden und umfaßt somit die Wirkung auf mehrere Generationen. $\mathrm{Zu}$ bestimmen sind die Konzentrationen der Prüfsubstanz, die im Vergleich zur Kontrolle zu einer $50 \%$ igen Abnahme entweder des Wachstums $\left(E_{b} C 50\right)$ oder der Wachstumsrate $\left(E_{r} C 50\right)$ führen sowie die NOEC. Eingesetzt werden mindestens 5 Konzentrationen in geometrischer Reihe bei einem Konzentrationsverhältnis, das nicht über 2,2 liegen darf; ein LimitTest mit $100 \mathrm{mg} / \mathrm{l}$, oder im Bereich der Wasserlöslichkeit ohne Lösungsvermittler, ist möglich.

Die analytische Bestimmung der Prüfsubstanz zur Überprüfung der Qualitätskriterien (mindestens 80 \%iger Erhalt der Ausgangskonzentration) direkt aus dem Versuchsgefäß ist u.a. wegen einer möglichen Adsorption der Substanz an den Algen häufig weder sinnvoll noch durchführbar. In diesem Fall wird ein Parallelansatz ohne Algen empfohlen.

\subsubsection{Terrestrische Toxizität}

\section{Akute Toxizität am Regenwurm (OECD 207)}

Der Test wird mit Eisenia foetida durchgeführt; zu bestimmen ist die Letalität innerhalb eines Zeitraums von 72 Stunden in Form des LC50-Wertes. Der Versuch kann als
Filterpapier-Test oder in einer künstlichen Erde durchgeführt werden.

\section{Wachstumshemmung an höheren Pflanzen (OECD 208)}

Aus drei Gruppen von höheren Pflanzen (Einkeimblättrige, Zweikeimblättrige, Leguminosen) wird jeweils mindestens ein Vertreter ausgewählt. Bestimmt werden der EC50-Wert - das ist in diesem Falle die Konzentration, die eine $50 \%$ ige Veränderung des Wachstumsverhaltens im Vergleich zur Kontrolle bewirkt - und der LC50-Wert, der sich auf das sichtbare Erscheinen der Keimlinge an der Bodenoberfläche bezieht.

\subsection{Toxizitätstests der Stufe II}

Im Gegensatz zur Grundstufe und zur Stufe I sind die Tests zur Stufe II ChemG nicht festgelegt. Das Prüfprogramm wird unter Berücksichtigung der bereits vorhandenen Kenntnisse in Diskussion zwischen Anmeldern und Zulassungsbehörden aufgestellt. Im folgenden sind einige Möglichkeiten der Stufe II-Tests aufgeführt:

- Life-Stage-Test am Fisch

- Test zur Embryotoxizität und Teratogenität am Krallenfrosch

- Akute Toxizität an Springschwänzen

- Akute Toxizität und Reproduktionstoxizität an Enchyträen

- Akute Toxizität an Nutzinsekten (Bienen, Florfliegen, Marienkäfer, Raubmilben, Laufkäfer)

- Subakute Toxizität/Reproduktion an Florfliegen

- Akute Toxizität an Sedimentorganismen

- Vogeltoxizität: akut, subakut, Reproduktion

- Wachstumshemmung an der Wasserlinse

- Test an Tetrabymena: Wachstum, Chemotaxis

- Test in aquatischen und terrestrischen Ökosystemen.

\section{Biologische Abbaubarkeit}

Der biologische Abbau ist einer der wichtigsten Prozesse zur Minderung des Gefährdungspotentials einer in die Umwelt gelangenden Substanz, ohne den die Abschätzung einer realistischen Umweltkonzentration nicht möglich ist. Daher wird bei der Anmeldung eines neuen Stoffes nach dem Chemikaliengesetz die biologische Abbaubarkeit geprüft, wobei von dem Grundsatz ausgegangen wird, daß mit der Produktionsmenge i.a. auch das Gefährdungspotential ansteigt. Der Prüfumfang ist folglich nach Produktionsmengen gestaffelt, die 3 Stufen zugeordnet sind:

\subsection{Biologische Abbaubarkeit in der Grundstufe}

Auf der Grundstufe werden Chemikalien mittels international standardisierter Tests (OECD- bzw. EG-Richtlinien) auf biologisch leichte Abbaubarkeit geprüft. Tabelle 1 gibt eine Übersicht der Prüfverfahren und deren Anwendbarkeit (gemäß Novellierung zur 7. Änderungsrichtlinie, EG, Stand 1992) ( $\rightarrow$ Tabelle 1).

Je nach Richtlinie wird als Prüfparameter gemessen:

- die Abnahme des gelösten organischen Kohlenstoffs der Testsubstanz,

- die durch den Abbau verursachte Sauerstoffabnahme,

- das dabei entstehende Kohlendioxid.

Diese Summenparameter sind unter den Testbedingungen 
Tabelle 1: Übersicht der Prüfverfahren der Grundstufe und deren Anwendbarkeit

\begin{tabular}{|c|c|c|c|c|c|}
\hline \multirow[b]{2}{*}{ EG-RL-Methode } & \multirow[b]{2}{*}{ Prüfverfahren } & \multirow[b]{2}{*}{ Analysenmethode } & \multicolumn{3}{|c|}{ Eignung für folgende Substanzen: } \\
\hline & & & schwer löslich & flüchtig & adsorbierend \\
\hline C. $4-\mathrm{A}$ & $\begin{array}{l}\text { DOC-Die Away } \\
\text { Test }\end{array}$ & $\begin{array}{l}\text { Gelöster org. } \\
\text { Kohlenstoff }\end{array}$ & - & - & $+1-$ \\
\hline C.4-B & $\begin{array}{l}\text { Mod. OECD- } \\
\text { Screening-Test }\end{array}$ & $\begin{array}{l}\text { Gelöster org. } \\
\text { Kohlenstoff }\end{array}$ & - & - & $+l-$ \\
\hline C. $4-\mathrm{C}$ & $\begin{array}{l}\mathrm{CO}_{2} \text {-Entwicklungs- } \\
\text { test (Mod. } \\
\text { Sturm-Test) }\end{array}$ & $\begin{array}{l}\text { Respirationstest: } \\
\mathrm{CO}_{2} \text {-Entwicklung }\end{array}$ & + & - & + \\
\hline C.4-D & $\begin{array}{l}\text { Manometr. Respi- } \\
\text { rationstest }\end{array}$ & $\begin{array}{l}\text { Manometrische } \\
\text { Messung: Sauer- } \\
\text { stoffverbrauch }\end{array}$ & + & $+1-$ & + \\
\hline C. $4-E$ & $\begin{array}{l}\text { Geschloss. } \\
\text { Flaschentest }\end{array}$ & $\begin{array}{l}\text { Respirationstest: } \\
\text { Sauerstoffver- } \\
\text { brauch }\end{array}$ & $+1-$ & + & + \\
\hline C. $4-F$ & MITI-Test (1) & $\begin{array}{l}\text { Respirationstest: } \\
\text { Sauerstoffver- } \\
\text { brauch }\end{array}$ & + & $+1-$ & + \\
\hline
\end{tabular}

Indizien für vollständigen Abbau. Der biologische Abbau erfolgt durch nicht adaptierte Mikroorganismen aus kommunalen Kläranlagen, die in entsprechender Menge zugesetzt werden.

Wird im Verlauf von 28 Tagen unter den relativ ungünstigen Testbedingungen ein Abbau erzielt, bei dem die Abnahme des gelösten organischen Kohlenstoffs $70 \%$ und mehr beträgt, bzw. bei dem mehr als $60 \%$ des ursprünglich vorhandenen Sauerstoffs verbraucht oder mehr als $60 \%$ Kohlendioxid produziert wurde, kann davon ausgegangen werden, daß die Substanz auch in der Umwelt problemlos und vollständig abgebaut werden kann.

Erfolgt der Abbau in dieser Zeitspanne nicht nur vollständig, sondern auch - nachdem er nach einer mehr oder weniger langen Anwachsphase der Mikroorganismen erst einmal eingesetzt hatte - in einem kurzen Zeitintervall, erhält die Substanz das Prädikat „biologisch leicht abbaubar" ${ }^{\star}$. Definiert wird dieser Begriff durch das sog. „10-Tage Fenster": das Abbauziel, d.h. $70 \%$ bzw. $60 \%$ Abbau, muß binnen 10 Tagen nach Überschreiten der $10-\%$ Schwelle erreicht werden. Nur unter dieser Voraussetzung, um die es international viele Diskussionen gab, darf eine Substanz als „biologisch leicht abbaubar" eingestuft werden.

Abb. 2 erläutert das Konzept des 10-Tage-Fensters am Beispiel einer idealisierten Abbaukurve $(\rightarrow A b b .2)$.

Im vorliegenden Fall, in dem der Abbau durch die Abnahme von gelöstem organischen Kohlenstoff $(\mathrm{DOC}=$ Dissolved Organic Carbon) charakterisiert sein soll, wurde nach Überschreiten der 10-\%-Schwelle am 9. Tag innerhalb von 10 Tagen bis zum 19. Tag ein Abbau von $75 \%$ und bis zum 28 . Tag von $87 \%$ erzielt.

Die Kriterien ( $70 \%$ Abnahme in 28 Tagen) und die Bedingungen des 10-Tage-Fensters sind somit eingehalten: Die Substanz darf als "biologisch leicht abbaubar" eingestuft werden.

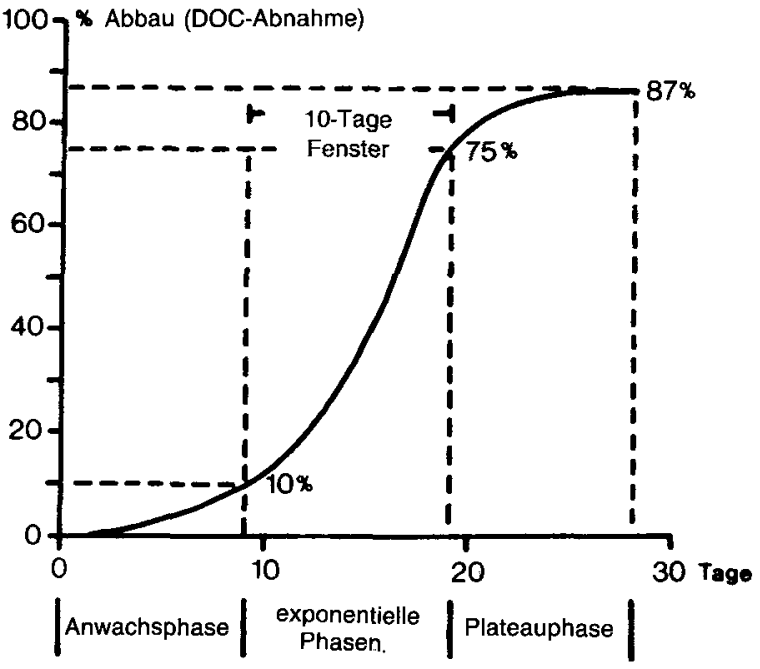

Abb. 2: Idealisierte Abbaukurve und Konzept des 10-Tage-Fensters

Wird das Abbauziel nach 28 Tagen nicht erreicht oder wird das 10-Tage-Fenster nicht eingehalten, wird die Substanz zunächst als „biologisch nicht leicht abbaubar" eingestuft.

\subsection{Biologische Abbaubarkeit in der Stufe 1}

Auf Stufe 1 werden weitere Prüfungen notwendig, die eine Entscheidung darüber zulassen, ob und bis zu welchem Grad eine Chemikalie potentiell abbaubar bzw. eliminierbar ist.

Die Bedingungen in diesen Untersuchungen sind für biologische Abbauvorgänge wesentlich günstiger als in den Tests auf leichte Abbaubarkeit. 


\section{Prüfverfahren der Stufe 1:}

- Zahn-Wellens-Test (L 133/99, Anhang V)

- Modifizierter S.C.A.S.-Test (L 133/123, Anhang V) (Semicontinuous Activated Sludge)

Prüfparameter in beiden Tests ist die DOC-Abnahme, der neben mikrobiellen Abbauprozessen auch abiotische Prozesse wie Adsorption und Verflüchtigung zugrunde liegen können. Da die Versuchsbedingungen teilweise erheblich von realen Umweltbedingungen abweichen, ist eine Übertragung der Ergebnisse auf die Umwelt nur sehr bedingt möglich; vielmehr wird aufgezeigt, ob die Substanz unter bestimmten Bedingungen überhaupt abbaubar ist.

Die Einstufung auf der Basis der DOC-Abnahme wird folgendermaßen vorgenommen:

\section{DOC-Abnahme}

$<20 \%$ nicht abbaubar/eliminierbar

$20-70 \%$ potentiell abbaubar/eliminierbar

$>70 \%$ Hinweis auf vollständigen Abbau/Eliminationen

Prüfungen auf Stufe 1 (u.U. auch Stufe 2) zum biologischen Abbau können bei Erreichen von $10 \mathrm{t} / \mathrm{a}$ auch bei besonderer ökotoxikologischer Indikation erforderlich werden. Diese liegt vor bei

- hoher akuter aquatischer Toxizität,

- Hinweis auf Bioakkumulationspotential $\left(\log _{P_{\text {ow }}}>2.7\right)$,

- biologisch nicht leichter Abbaubarkeit.

Für Substanzen, die schon unter den strengen Bedingungen der Grundstufentests als biologisch leicht abbaubar eingestuft wurden, entfallen zunächst auf Stufe 1 weitere Prüfungen. Nur bei Vermarktungsmengen $>1000 \mathrm{t} / \mathrm{a}$ wird mittels Prüfung der Stufe 2 überprüft, ob das Abbauergebnis auch unter umweltrelevanten Bedingungen gültig ist.

\subsection{Biologische Abbaubarkeit in der Stufe 2}

Auf Stufe 2 wird zur Abschätzung des Abbauverhaltens einer Chemikalie in der Umwelt ein Simulationstest erforderlich. Dazu ist für den Bereich Kläranlagen im Anhang V der Richtlinie 79/831/EWG der Simulationstest mit Belebtschlamm (L 133/106) vorgesehen; jedoch können je nach Erfordernis im Dialog mit dem Anmelder Simulationstests auch für andere Umweltbereiche, z.B. Oberflächengewässer, durchgeführt werden.

\section{Bioakkumulation}

Auf der Basis des Verteilungskoeffizienten Octanol/Wasser $\left(\mathrm{P}_{\text {ow }}\right)$, zu dem Angaben im Grunddatensatz einer Anmeldung nach dem ChemG gemacht werden, lassen sich aufgrund eines linearen Zusammenhangs mit der Bioakkumulation in aquatischen Organismen gewisse Voraussagen über deren Höhe machen. Dieses Abschätzverfahren wird als erster Schritt innerhalb eines Stufenplans zur Ermittlung der Bioakkumulation angewandt.

\subsection{Bioakkumulation in der Grundstufe}

Anhand des $\log \mathrm{P}_{\mathrm{ow}}$-Wertes wird ein Bioakkumulationspotential abgeschätzt. Nimmt man als Schwellenwert zu einer nennenswerten Bioakkumulation einen Biokonzentrationsfaktor (BCF) von 50, dann entspricht diesem Wert erfahrungsgemäß in grober Näherung ein $\log \mathrm{P}_{\text {ow }}$ von 2.7 .

Weist eine Chemikalie einen $\log \mathrm{P}_{\text {ow }}>2.7$ auf, so lautet die Einstufung:

Hinweis auf Bioakkumulationspotential.

Liegt der $\log P_{\text {ow }}$ unter 2.7, so lautet die Einstufung:

Kein Hinweis auf Bioakkumulation.

Bis zu einem log $P_{\text {ow }}$ von 6 steigt die Anreicherung linear an; darüber nimmt sie jedoch mit sinkender Bioverfügbarkeit nicht mehr in demselben Maße zu, so daß oberhalb einer gewissen Molekülgröße und -form eine Bioakkumulation immer unwahrscheinlicher wird:

Die Wahrscheinlichkeit einer Akkumulation bei einem log $\mathrm{P}_{\mathrm{ow}}>6$ in Verbindung mit einem Molekulargewicht $>$ 600 ist gering.

Stoffe mit oberflächenaktiven und weiteren spezifischen Eigenschaften - also vor allen Dingen Tenside - weichen von diesem Schema $\mathrm{ab}$, da hier selbst bei $\log \mathrm{P}_{\mathrm{ow}}<2.7$ eine signifikante Bioakkumulation/Biosorption möglich ist.

\subsection{Bioakkumulation in der Stufe 1}

Unabhängig von der Höhe des $\log \mathrm{P}_{\mathrm{ow}}$ ist bei einer Vermarktungsmenge von $100 \mathrm{t} / \mathrm{a}$ bzw. $500 \mathrm{t}$ kumulativ eine Untersuchung zur Bioakkumulation erforderlich.

Dazu stehen z.Zt. 5 gleichwertige OECD-Richtlinien (305 $A-E)$ zur Verfügung:

305 A Sequentieller Fischtest

305 B Semistatischer Fischtest

$305 \mathrm{C}$ Test zur Ermittlung der Biokonzentration

305 D Statischer Fischtest

305 E Flow-Through-Fischtest

$$
\begin{aligned}
& \text { statisch } \\
& \text { semi-statisch } \\
& \text { dynamisch } \\
& \text { statisch } \\
& \text { dynamisch }
\end{aligned}
$$

Von diesen wird vorzugsweise der OECD 305 E FlowThrough-Fischtest durchgeführt, weil er neben der Höhe der Bioakkumulation auch Angaben zur Ausscheidung der Substanz liefert.

Zur Bewertung der Bioakkumulation hat das Umweltbundesamt ein Konzept entwickelt, das auf folgenden BasisKriterien beruht:

- Biokonzentrationsfaktor BCF

- Ausscheidungsrate $\mathrm{Ct} 50$ (Clearance time für $50 \%$ Elimination)

Diese beiden Kriterien, die jeweils in 4 Klassen eingeteilt werden, gehen gleichwertig in eine Mittelwertbildung ein, die zu 4 Bewertungsklassen der Bioakkumulation führt $(\rightarrow$ Tabellen $2-4)$.

\section{Beispiel:}

Ein Stoff mit einem BCF von 300 (Klasse III) und einer Ct50 von $<3$ Tagen (Klasse I) hat bei der Mittelwertbildung eine Bioakkumulations-Klasse von II, d.h. er zeigt eine kurzfristig hohe BA. Damit ist ein Hinweis auf ein Risikopotential gegeben. 
Tabelle 2: Klassifizierung der Bioakkumulationsfaktoren (BCF)

\begin{tabular}{lll}
\hline BCF & Klasse & Bewertung \\
\hline$<30$ & I & schwach \\
$30-100$ & II & mäBig \\
$100-1000$ & III & hoch \\
$>1000$ & IV & sehr hoch \\
\hline
\end{tabular}

Tabelle 3: Klassifizierung der Ausscheidung

\begin{tabular}{|c|c|c|}
\hline CT50 & Klasse & Bewertung \\
\hline $\begin{array}{l}<3 \text { Tage } \\
3-10 \text { Tage }\end{array}$ & $\begin{array}{l}\text { I } \\
\text { II }\end{array}$ & $\begin{array}{l}\text { schnell } \\
\text { verzögert } \\
\text { (kurzfristige BA) }\end{array}$ \\
\hline $10-30$ Tage & III & $\begin{array}{l}\text { langsam } \\
\text { (mittelfristige BA) }\end{array}$ \\
\hline$>30$ Tage & IV & $\begin{array}{l}\text { unbedeutend } \\
\text { (langfristige BA) }\end{array}$ \\
\hline
\end{tabular}

$\mathrm{BA}=$ Bioausscheidung

Tabelle 4: Gesamtbewertung der Bioakkumulation (Kombination aus Tabelle 2 und 3 )

\begin{tabular}{ll}
\hline Klasse & Bewertung \\
\hline I & kein AnlaB zur Besorgnis \\
II & $\begin{array}{l}\text { Hinweis auf Risikopotential } \\
\text { III }\end{array}$ \\
AnlaB zur Besorgnis; ggf. Eingriffsschwelle für län- \\
gerfristige ökotoxikologische Prüfungen \\
unvertretbares Risiko
\end{tabular}

\section{Bewertung und vorgezogene Prüfungen}

Wie in den Kapiteln 3 und 4 ausgeführt wurde, erlauben die Grundstufendaten eine Unterscheidung zwischen einer leichten und nicht leichten biologischen Abbaubarkeit der Prüfsubstanz sowie eine Abschätzung des Bioakkumulationspotentials. Bei einer besonderen ökotoxikologischen
Indikation, also kleinen LC50-Werten für Fische bzw. kleinen EC50-Werten für Daphnien, nicht leichter biologischer Abbaubarkeit und einem $\log \mathrm{P}_{\text {ow }}$-Wert $>2.7$ kann eine genauere Abklärung des Bioakkumulationspotentials bereits auf der Grundstufenebene notwendig werden. Entscheidend für eine Forderung nach vorgezogenen Tests der Stufe 1 auf der Grundstufenebene ist generell der Vergleich der ökotoxikologischen Daten mit den prognostizierten maximalen Konzentrationen des Stoffes in der Umwelt. Umweltgefährlich - und somit nach ChemG zu regulieren - ist ein Stoff, dessen Wirkdaten gleich oder kleiner der zu erwartenden Umweltkonzentration sind. Der Verdacht auf eine Umweltgefährlichkeit besteht, wenn die zu erwartende Konzentration in Oberflächengewässern unwesentlich geringer ist als die beginnende Wirkkonzentration. Für diesen Fall werden zur Abklärung des Verdachts sofort vorgezogene ökotoxikologische Prüfungen der Stufe 1 verlangt.

Die genaue Vorgehensweise bei der Forderung vorgezogener Prüfungen ist in der UBA-TEXTE $28 / 90$ beschrieben.

\section{Literatur}

NAGEL, R.; R. LoSkill (Ed.): Bioaccumulation in Aquatic Systems. Contributions to the Assessment Proceedings of an International Workshop, Berlin 1990. VCH Verlagsgesellschaft mbH, Weinheim 1991

Richtlinie 92/32/EWG des Rates vom 30. April zur 7. Änderung der Richtlinie 67/548/EWG zur Angleichung der Rechts- und Verwaltungsvorschriften für die Einstufung, Verpackung und Kennzeichnung gefährlicher Stoffe, Amtsblatt der Europäischen Gemeinschaften, im Druck

Steinhäuser, K.G.: Prüfung schwerlöslicher Substanzen in aquatischen Toxizitätstests. Schr. Reihe Verein WaBoLu, Gustav Fischer Verlag, Stuttgart, im Druck

UBA-TEXTE 28/90: Chemikaliengesetz, Heft 9. Grundzüge der Bewertung von Neuen Stoffen nach dem ChemG - 1. Fortschreibung 1990

UBA-TEXTE 42/91: Bioakkumulation - Bewertungskonzept und Strategien im Gesetzesvollzug. 1991

UBA-TEXTE: Biologischer Abbau - Bewertungskonzept und Strategien im Gesetzesvollzug. 1993, im Druck

Glossar

DOC: Dissolved Organic Carbon

Gehalt an gelöstem organischen Kohlenstoff (Bestimmung nach Filtration der Probe).

EC: Effektive (wirksame) Konzentration (Effective Concentration)

Die $\mathrm{EC}_{0}$ ist die höchste getestete Konzentration, bei der keine Wirkung gegenüber den Testorganismen beobachtet wird, die $\mathrm{EC}_{100}$ die niedrigste getestete Konzentration, bei der alle Testorganismen und zu Vergleichszwecken die $\mathrm{EC}_{50}$, bei der $50 \%$ der Testorganismen betroffen sind. Die $\mathrm{EC}_{50}$ wird mit geeigneten statistischen Verfahren bestimmt.

LC: Letale Konzentration (Lethal Concentration; siehe analog LD)

LD: Letaldosis

$\mathrm{LD}_{100}$ ist die gegenüber allen Testorganismen tödliche Dosis, $\mathrm{LD}_{50}$ die Dosis letalis media (mitrlere tödliche Dosis), bei der $50 \%$ der Versuchstiere getötet werden. Gebräuchlich ist auch die $\mathrm{LD}_{0}$, die maximale Dosis, die sich als nicht letal gegenüber den Testorganismen erweist.

$\mathrm{E}_{\mathrm{b}} \mathrm{C}_{50}: \quad 50 \%$ ige Abnahme des Wachstums

$\mathrm{E}=$ Effekte

b $=$ Biomasse

$\mathrm{E}_{r} \mathrm{C}_{50}: \quad 50 \%$ ige Abnahme der Wachstumsrate

$\mathrm{r}=$ Geschwindigkeitsrate

$\mathrm{Ct}_{50}: \quad$ Ausscheidungsrate (Clearance time für $50 \%$ Elimination) 